PROCEEDINGS OF THE

AMERICAN MATHEMATICAL SOCIETY

Volume 136, Number 9, September 2008, Pages 3089-3094

S 0002-9939(08)09296-4

Article electronically published on April 30, 2008

\title{
ON THE SIGN CHANGES OF COEFFICIENTS OF GENERAL DIRICHLET SERIES
}

\author{
WLADIMIR DE AZEVEDO PRIBITKIN
}

(Communicated by Ken Ono)

\begin{abstract}
Under what conditions do the (possibly complex) coefficients of a general Dirichlet series exhibit oscillatory behavior? In this work we invoke Laguerre's Rule of Signs and Landau's Theorem to provide a rather simple answer to this question. Furthermore, we explain how our result easily applies to a multitude of functions.
\end{abstract}

\section{INTRODUCTION}

Let $\left\{a_{n}\right\}_{n=1}^{\infty}$ be any nontrivial sequence of complex numbers. If the numbers are all real, then it is natural to ask whether the sequence is oscillatory. This means that there exist infinitely many $n$ such that $a_{n}>0$ and infinitely many $n$ such that $a_{n}<0$. To accommodate the possibility that the numbers are not all real, we introduce a generalized notion. We call the sequence $\left\{a_{n}\right\}_{n=1}^{\infty}$ oscillatory if, for each real number $\phi \in[0, \pi)$, either the sequence $\left\{\operatorname{Re}\left(e^{-i \phi} a_{n}\right)\right\}_{n=1}^{\infty}$ is oscillatory or is trivial. Geometrically, this means simply that no matter how we slice the plane with a straight line going through the origin, there will always be infinitely many terms of the sequence on either side of the line, unless all of the terms are on the line itself. In the latter case, which can happen for at most one slice, there must be infinitely many terms on either open ray emanating from the origin.

In this note we harness the generating power of a general Dirichlet series to supply a simple condition which guarantees that a sequence is oscillatory. Specifically, we show the following:

Theorem 1. Let $F(s)=\sum_{n=1}^{\infty} \frac{a_{n}}{e^{\lambda_{n} s}}$ be a nontrivial general Dirichlet series which converges somewhere. Here the coefficient sequence $\left\{a_{n}\right\}_{n=1}^{\infty}$ is complex and the exponent sequence $\left\{\lambda_{n}\right\}_{n=1}^{\infty}$ is real and strictly increasing to $\infty$. If the function $F$ is holomorphic on the whole real line and has infinitely many real zeros, then $\left\{a_{n}\right\}_{n=1}^{\infty}$ is oscillatory.

As usual, here and throughout, 'the function $F$ ' refers to the analytic continuation (assuming it exists) of the function defined originally by the series. In the next section we prove Theorem 1 , and in the last section we demonstrate its wide applicability.

Received by the editors July 23, 2007, and, in revised form, July 29, 2007.

2000 Mathematics Subject Classification. Primary 11M41, 30B50.

Key words and phrases. General Dirichlet series, oscillatory sequence, Mellin transform.

This work was supported (in part) by The City University of New York PSC-CUNY Research Award Program (grant \#68327-00 37).

(C)2008 American Mathematical Society 


\section{Proof of Theorem 1}

The proof relies upon the following two important results:

Laguerre's Rule of Signs. Let $F(s)=\sum_{n=1}^{\infty} \frac{a_{n}}{e^{\lambda_{n}} s}$, with $\left\{\lambda_{n}\right\}_{n=1}^{\infty}$ real and strictly increasing to $\infty$, be a nontrivial general Dirichlet series which converges somewhere. If the coefficient sequence $\left\{a_{n}\right\}_{n=1}^{\infty}$ is real and has finitely many sign changes, then the number of real zeros of $F$ in the interior of its domain of convergence is less than or equal to the number of sign changes of $\left\{a_{n}\right\}_{n=1}^{\infty}$.

This result, a little-known generalization of Descartes's classic rule, was provided in 1883 by Laguerre [8]. His proof uses Rolle's Theorem and induction on the number of sign changes. Note that the zeros are counted according to their multiplicity. (For similar statements and some extensions, consult [10, pp. 46-48].)

Landau's Theorem. Let $F(s)=\sum_{n=1}^{\infty} \frac{a_{n}}{e^{\lambda_{n} s}}$, with $\left\{\lambda_{n}\right\}_{n=1}^{\infty}$ real and strictly increasing to $\infty$, be a general Dirichlet series which converges somewhere, but not everywhere. If the coefficient sequence $\left\{a_{n}\right\}_{n=1}^{\infty}$ is real and has finitely many sign changes, then the function $F$ has a singularity at the real point of its line of convergence.

This theorem, given by Landau 9] in 1905, is rather well known. (For the proof, see [5, p. 10] or [11, pp. 67-68].)

We are now ready to prove Theorem 1 . Let's first consider the special case where the coefficient sequence $\left\{a_{n}\right\}_{n=1}^{\infty}$ is real. By way of contradiction, suppose that it is not oscillatory, in other words, that it has finitely many sign changes. There are just two possibilities: (i) $F$ converges everywhere, but is nontrivial; or (ii) $F$ converges somewhere, but not everywhere. In the first scenario, we know by Laguerre's Rule of Signs that the total number of real zeros of $F$ is finite. This negates the hypothesis that $F$ possesses infinitely many real zeros. In the second scenario, we see by Landau's Theorem that $F$ must have a singularity at the real point of its line of convergence. This contradicts the assumption that $F$ is holomorphic on the real axis. Either way, we have established Theorem 1 for $\left\{a_{n}\right\}_{n=1}^{\infty}$ real.

We next examine the general case where the coefficient sequence $\left\{a_{n}\right\}_{n=1}^{\infty}$ need not be real. Let $\phi \in[0, \pi)$, and consider the auxiliary function

$$
G_{\phi}(s)=\frac{e^{-i \phi} F(s)+\overline{e^{-i \phi} F(\bar{s})}}{2}=\sum_{n=1}^{\infty} \frac{\operatorname{Re}\left(e^{-i \phi} a_{n}\right)}{e^{\lambda_{n} s}} .
$$

Clearly, the function $G_{\phi}$ is holomorphic on the real line, where it also has infinitely many zeros. Hence, for each $\phi$ we must have that either the sequence $\left\{\operatorname{Re}\left(e^{-i \phi} a_{n}\right)\right\}_{n=1}^{\infty}$ is oscillatory or is trivial. This means that $\left\{a_{n}\right\}_{n=1}^{\infty}$ is oscillatory and secures Theorem 1 altogether.

\section{EXAMPLES AND COMMENTS}

We sketch some fundamental examples to illustrate the utility of Theorem 1. For succinctness, we shall label the function defined originally by a nontrivial general Dirichlet series as EZ if it is entire and has infinitely many real zeros.

(1) Suppose $f$ is a nontrivial complex-valued continuous function on the half-line $\mathbb{R}^{+}=(0, \infty)$ that is $O\left(y^{-N}\right)$ as $y \rightarrow \infty$ and $O\left(y^{N}\right)$ as $y \rightarrow 0$, for every nonnegative 
integer $N$. Since $f$ is "rapidly decreasing" as $y \rightarrow \infty$ and as $y \rightarrow 0$, its Mellin transform

$$
M\{f ; s\}=\int_{0}^{\infty} f(y) y^{s-1} d y
$$

converges everywhere and is clearly entire. Furthermore assume that $f$ possesses an expansion of the type

$$
f(y)=\sum_{n=1}^{\infty} a_{n} h\left(e^{\lambda_{n}} y\right), \quad y>0
$$

where $\left\{a_{n}\right\}_{n=1}^{\infty}$ is complex and $\left\{\lambda_{n}\right\}_{n=1}^{\infty}$ is real and strictly increasing to $\infty$. Here $h$ is some complex-valued continuous function on $\mathbb{R}^{+}$that is rapidly decreasing as $y \rightarrow \infty$ and $O\left(y^{c}\right)$ as $y \rightarrow 0$, for some real number $c$. Next, consider the general Dirichlet series

$$
F(s)=\sum_{n=1}^{\infty} \frac{a_{n}}{e^{\lambda_{n} s}}
$$

and suppose it converges somewhere. An easy calculation tells us that

$$
M\{f ; s\}=M\{h ; s\} F(s)
$$

for $\operatorname{Re}(s) \gg 0$ (in fact, wherever $F$ converges and $\operatorname{Re}(s)>-c$ ). Observe that $M\{h ; s\}$, the Mellin transform of $h$, is analytic for $\operatorname{Re}(s)>-c$. Now, if the reciprocal of $M\{h ; s\}$ admits an analytic continuation to an entire function that has infinitely many real zeros, then obviously $F$ is EZ, and therefore by Theorem $1\left\{a_{n}\right\}_{n=1}^{\infty}$ is oscillatory.

We remark that if the growth condition on $f$ as $y \rightarrow 0$ is relaxed, then a more involved situation ensues. Let's assume, for example, that (an extension of) $f$ is in the Schwartz space of $\mathbb{R}$. (Recall that this consists of the infinitely differentiable functions which together with their derivatives are rapidly decreasing as $|y| \rightarrow \infty$.) Then $M\{f ; s\}$ is analytic for $\operatorname{Re}(s)>0$ and (thanks to integration by parts) admits a meromorphic continuation to all of $\mathbb{C}$ with at most simple poles at the nonpositive integers. In fact, $M\{f ; s\}$ is analytic at $s=-m, m \geq 0$, if and only if $f^{(m)}(0)=0$. If we retain the assumption that $1 / M\{h ; s\}$ extends to an entire function with infinitely many real zeros, then it is clear that $F$ has a meromorphic continuation to all of $\mathbb{C}$ with at most simple poles at the nonpositive integers. Furthermore, $F$ is analytic at $s=-m$ if and only if $f^{(m)}(0)=0$ or $1 / M\{h ;-m\}=0$. So $F$ is entire if and only if $f^{(m)}(0) / M\{h ;-m\}=0$ for all $m \geq 0$. Now, $F$ is EZ if it's entire and either $f^{(m)}(0)=1 / M\{h ;-m\}=0$ for infinitely many $m$ or if infinitely many of the real zeros of $1 / M\{h ; s\}$ survive to be zeros of $F$. Under such favorable conditions, it follows by Theorem 1 that $\left\{a_{n}\right\}_{n=1}^{\infty}$ is oscillatory.

What does $h$ look like? Note that under the stipulations stated in the last sentence of the first paragraph of (1), $h$ is neither of compact support on $\mathbb{R}^{+}$ nor rapidly decreasing as $y \rightarrow 0$. (But if $c=0$, then (an extension of) $h$ could conceivably be in the Schwartz space of $\mathbb{R}$.) Some candidates for $h$ are afforded by the prototypical example of the decaying exponential function

$$
e^{-y}, \quad y>0,
$$

by the generalized gamma function

$$
\Gamma_{y}(\alpha)=\int_{0}^{\infty} t^{\alpha-1} e^{-t-y / t} d t, \quad y>0, \alpha \in \mathbb{C},
$$


and by the extended generalized gamma function

$$
\Gamma_{\nu}(\alpha ; y)=\left(\frac{2 y}{\pi}\right)^{1 / 2} \int_{0}^{\infty} t^{\alpha-3 / 2} e^{-t} K_{\nu+1 / 2}(y / t) d t, \quad y>0, \alpha \in \mathbb{C}, \nu \in \mathbb{C} .
$$

Here $K$ is the modified Bessel function of the second kind (also called the Macdonald function), and in fact, $\Gamma_{y}(\alpha)=2 y^{\alpha / 2} K_{\alpha}(2 \sqrt{y})$. Note that $\Gamma_{0}(\alpha ; y)=\Gamma_{y}(\alpha)$. It is rather well known that

$$
M\left\{e^{-y} ; s\right\}=\Gamma(s)
$$

for $\operatorname{Re}(s)>0$, and easy to verify that

$$
M\left\{\Gamma_{y}(\alpha) ; s\right\}=\Gamma(s) \Gamma(s+\alpha)
$$

if $\operatorname{Re}(s)>0$ and $\operatorname{Re}(s+\alpha)>0$, and

$$
M\left\{\Gamma_{\nu}(\alpha ; y) ; s\right\}=\pi^{-1 / 2} 2^{s-1} \Gamma\left(\frac{s-\nu}{2}\right) \Gamma\left(\frac{s+\nu+1}{2}\right) \Gamma(s+\alpha)
$$

if $\operatorname{Re}(s+1 / 2)>|\operatorname{Re}(\nu+1 / 2)|$ and $\operatorname{Re}(s+\alpha)>0$. In all three cases the reciprocal of the Mellin transform is entire in $s$ and (assuming $\alpha$ or $\nu$ is real for the last case) has infinitely many real zeros. What's more, by repeatedly invoking the key convolution property [4, p. 308]

$$
M\left\{\int_{0}^{\infty} t^{\alpha-1} h_{1}(t) h_{2}(y / t) d t ; s\right\}=M\left\{h_{1}(y) ; s+\alpha\right\} M\left\{h_{2}(y) ; s\right\}
$$

for two suitable functions $h_{1}$ and $h_{2}$ on $\mathbb{R}^{+}$, it is not hard to churn out infinitely many more such functions. For additional information about the aforementioned gamma functions, see [3], and for an interesting discussion concerning Mellin transforms, read [2, pp. 106-114].

(2) Let $F(s)=\sum_{n=1}^{\infty} \frac{a_{n}}{e^{\lambda_{n} s}}$ and $G(s)=\sum_{m=1}^{\infty} \frac{b_{m}}{e^{\mu_{m} s}}$ be nontrivial general Dirichlet series which converge somewhere. As usual, the coefficient sequences $\left\{a_{n}\right\}_{n=1}^{\infty}$ and $\left\{b_{m}\right\}_{m=1}^{\infty}$ are complex and the exponent sequences $\left\{\lambda_{n}\right\}_{n=1}^{\infty}$ and $\left\{\mu_{m}\right\}_{m=1}^{\infty}$ are real and strictly increasing to $\infty$. Define the Dirichlet product of $F$ and $G$ by $H(s)=\sum_{\ell=1}^{\infty} \frac{c_{\ell}}{e^{\nu} \ell^{s}}$, where

$$
c_{\ell}=\sum_{\lambda_{n}+\mu_{m}=\nu_{\ell}} a_{n} b_{m}
$$

and $\left\{\nu_{\ell}\right\}_{\ell=1}^{\infty}$ is the ascending sequence formed by all the values of $\lambda_{n}+\mu_{m}$. Suppose that $H$ converges somewhere. It is known that this will be the case if at least one of $G$ or $H$ converges absolutely somewhere, or if $a_{n}=O\left(\frac{\lambda_{n}-\lambda_{n-1}}{\lambda_{n}}\right)$ and $b_{m}=O\left(\frac{\mu_{m}-\mu_{m-1}}{\mu_{m}}\right)$. Note that the former must happen if at least one of $F$ or $G$ is an ordinary Dirichlet series (or, more broadly, if at least one of $\lim \sup \frac{\log n}{\lambda_{n}}$ or $\lim \sup \frac{\log m}{\mu_{m}}$ is finite). Because $H$ converges somewhere, it follows that $H=F G$ wherever all three series converge. (For an explanation of all the above facts, see [5. p. 9 and pp. 62-66].) Now, assume that $F$ is $\mathrm{EZ}$ and that the function $G$ is entire. Then of course $H$ is itself EZ and so by Theorem $1\left\{c_{\ell}\right\}_{\ell=1}^{\infty}$ is oscillatory. More generally, suppose that both $F$ and $G$ have analytic continuations to functions that are meromorphic on the real line, that at least one of them possesses infinitely many real zeros, and that the real zeros and real poles cancel out in such a way that the function $H$ is holomorphic on the real line and has infinitely many real zeros. Then it is plain by Theorem 1 that $\left\{c_{\ell}\right\}_{\ell=1}^{\infty}$ is oscillatory. This formulation may seem rather obvious, and yet can be useful. For a simple example, 
let $F(s)=\sum_{n=1}^{\infty} \frac{a_{n}}{n^{s}}$ be a nontrivial ordinary Dirichlet series that converges somewhere, and let $G(s)=\frac{1}{\zeta(s)}$ be the reciprocal of the Riemann zeta-function. Then we have that the Dirichlet convolution

$$
c_{\ell}=\sum_{d \mid \ell, d>0} a_{d} \mu\left(\frac{\ell}{d}\right)
$$

where $\mu$ now denotes the Möbius function. If $F$ is EZ, say, with zeros at all of the nonpositive integers, then both $\left\{a_{n}\right\}_{n=1}^{\infty}$ and $\left\{c_{\ell}\right\}_{\ell=1}^{\infty}$ are oscillatory. For an even simpler example, let $F$ be any nontrivial ordinary Dirichlet polynomial that vanishes at $s=1$, and let $G(s)=\zeta(s)$.

(3) Is it possible for a general Dirichlet series to have infinitely many real zeros in the interior of its domain of convergence? The answer is yes, and there are two basic scenarios, both of which we illustrate briefly. First recall that if a general Dirichlet series converges somewhere, then it must be zero-free for all sufficiently large real values of $s$. (Also, if it converges absolutely somewhere, then it must be zero-free for all sufficiently large values of $\operatorname{Re}(s)$. For details on this, see [1, p. 227] as well as [5, p. 6].) Now let $f_{1}$ be any nontrivial entire function that has infinitely many positive real zeros, and consider its Maclaurin series $f_{1}(z)=\sum_{n=0}^{\infty} a_{n} z^{n}, z \in \mathbb{C}$. Then the general Dirichlet series $F_{1}(s)=f_{1}\left(e^{-s}\right)=\sum_{n=0}^{\infty} \frac{a_{n}}{e^{n s}}$, which is periodic with period $2 \pi i$, converges everywhere and possesses infinitely many negative real zeros. Next, let $f_{2}(z)=f_{1}\left(\frac{1}{1-z}\right)$ and consider its Maclaurin series $f_{2}(z)=\sum_{n=0}^{\infty} b_{n} z^{n},|z|<1$. Note that $f_{2}$ has infinitely many positive real zeros less than 1 and that they must accumulate at $z=1$, an essential singularity of $f_{2}$. Then the general Dirichlet series $F_{2}(s)=f_{2}\left(e^{-s}\right)=\sum_{n=0}^{\infty} \frac{b_{n}}{e^{n s}}$ converges for $\operatorname{Re}(s)>0$, where it has infinitely many positive real zeros. Of course, these zeros must accumulate at the origin, which must be an essential singularity for $F_{2}$. Clearly both $\left\{a_{n}\right\}_{n=0}^{\infty}$ and $\left\{b_{n}\right\}_{n=0}^{\infty}$ are oscillatory (by the "complexified" version of Laguerre's Rule of Signs). We remark that $\left\{\sum_{k=0}^{n} b_{k}\right\}_{n=0}^{\infty}$ is also oscillatory. (This follows immediately from considering $\left(1-e^{-s}\right)^{-1} F_{2}(s)$.) To see a concrete example of the above, the interested reader may examine, for instance, $f_{1}(z)=\sin \pi z$ or $f_{1}(z)=1 / \Gamma(1-z)$.

(4) Much of what we said has applications to arithmetic functions, particularly those connected with general $L$-functions and automorphic forms. We shall not pursue this here, except to say that all of the results pertaining to oscillatory behavior found in [6] and [7] can be established anew by applying Theorem 1. In a sequel we hope to address such matters further.

\section{ACKNOWLEDGMENT}

The author thanks the referee for a careful reading of the paper.

\section{REFERENCES}

[1] T.M. Apostol, Introduction to Analytic Number Theory, Undergraduate Texts in Math., corrected fifth printing, Springer-Verlag, New York, 1998. MR0434929 (55:7892)

[2] N. Bleistein and R. Handelsman, Asymptotic Expansions of Integrals, Dover, New York, 1986. MR863284(89d:41049)

[3] M.A. Chaudhry and S.M. Zubair, On a Class of Incomplete Gamma Functions with Applications, Chapman \& Hall/CRC, Boca Raton, FL. 2002. MR.1887130(2003c:33006)

[4] A. Erdélyi, W. Magnus, F. Oberhettinger, and F. Tricomi, Tables of Integral Transforms, 1, Bateman Manuscript Project, McGraw-Hill, New York, 1954. 
[5] G. H. Hardy and M. Riesz, The General Theory of Dirichlet's series, Stechert-Hafner, New York, 1964; reprint of first edition, Cambridge Univ. Press, Cambridge, 1915. MR0185094 $(32: 2564)$

[6] M. Knopp, W. Kohnen, and W. Pribitkin, On the Signs of Fourier Coefficients of Cusp Forms, Ramanujan J. 7 (2003), no. 3, 269-277. MR2035806 (2004m:11064)

[7] W. Kohnen, Sign Changes of Hecke Eigenvalues of Siegel Cusp Forms of Genus Two, Proc. Amer. Math. Soc. 135 (2007), no. 4, 997-999. MR2262899 (2007j:11057)

[8] E.N. Laguerre, Sur la Théorie des Équations Numériques, J. Math. Pures Appl. 9 (1883), 99-146 (also in Euvres de Laguerre, v. I, pp. 3-47, Gauthier-Villars, Paris, 1898).

[9] E. Landau, Über einen Satz von Tschebyschef, Math. Ann. 61 (1905), 527-550. MR1511360

[10] G. Pólya and G. Szegö, Problems and Theorems in Analysis II, Die Grundlehren der Mathematischen Wissenschaften 216, Springer-Verlag, Berlin, 1976. MR.1492448

[11] J-P. Serre, A Course in Arithmetic, Graduate Texts in Math. 7, corrected fourth printing, Springer-Verlag, New York, 1993. MR0344216 (49:8956)

Department of Mathematics, College of Staten Island, City University of New York, 2800 Victory Boulevard, Staten Island, New York 10314

E-mail address: pribitkin@mail.csi.cuny.edu

E-mail address: w_pribitkin@msn.com 